\title{
Mathematics for Industry in Europe
}

\author{
Wil Schilders (TU Eindhoven, The Netherlands)
}

In this paper ${ }^{1}$, we give an overview of the development of mathematics for industry in Europe. The advent of such activities was in the 1970s, when, especially in Oxford, the potential of applications of mathematics was realised by Alan Tayler and his colleagues, and the very successful study groups with industry were set up. It led to discussions about European organisations such as ECMI, founded in 1987, as well as to a number of reports on mathematics in industry, to commercial institutes exploiting mathematics for industrial applications and, finally, to a new organisation that has recently been founded, EU-MATHS-IN. It is important to share these experiences and activities with colleagues, anticipating that mathematics in industry will play a key role in enabling technology, leading, in many respects, to a better world, to innovations and solutions for the many challenges humanity is faced with..

\section{Introduction}

The mathematical sciences play a vital part in all aspects of modern society. Without research and training in mathematics, there would be no engineering, economics or computer science; no smart phones, MRI scanners, bank accounts or PIN numbers. Mathematics plays a key role in tackling the modern-day challenge of cyber security and in predicting the consequences of climate change, as well as in the manufacturing sectors of the automotive and aerospace industries through the utilisation of superior virtual design processes. Likewise, the life sciences sector, with significant potential for economic growth, would not be in such a strong position without mathematics research and training providing the expertise integral to the development of areas such as personalised healthcare and pharmaceuticals, as well as related medical technologies. The emergence of truly massive datasets across most fields of science and engineering increases the need for new tools from the mathematical sciences, combining traditional methods with artificial intelligence, machine learning and preparing for a future where high-performance computing will play a major role. Modelling, simulation and optimisation will need to be adapted to the data rich environments available nowadays, leading, for example, to major efforts in the area of digital twinning.

One of the classic ways in which mathematical science research plays a role in the economy is through the collection of data to help understand it and the use of tools and techniques to enable the discovery of new relationships or models. Modelling of physical phenomena already

\footnotetext{
1 This article is an adapted version of a keynote presentation given at the Forum "Math-for-Industry" 2013 - The Impact of Applications on Mathematics, November 4-8,2013, Fukuoka (Japan).
}

dates back several centuries, and well-known systems of equations with the names of Maxwell, Navier-Stokes, Korteweg-de Vries, and more recently the Schrödinger equation, plus many others, are now well established. But it was not until the advent of computers in the middle of the previous century and the development of sophisticated computational methods (like iterative solution methods for large sparse linear systems) that this could be taken to a higher level, by performing computations using these models. Software tools with advanced computational mathematical techniques for the solution of the aforementioned systems of equations have become commonplace and are heavily used by engineers and scientists.

Mirroring this activity is the increased awareness of society and industry that mathematical simulation is ubiquitous to addressing the challenging problems of our times. Industrial processes, economic models and critical events like floods, power failures or epidemics have become so complicated that their realistic description does not require the simulation of a single model, but rather the co-simulation of various models. Better scientific understanding of the factors governing these will provide routes to greater innovation power and economic well-being across an increasingly complex networked world with its competitive and strongly interacting agents. Industry, but also science, is highly dependent on the development of virtual environments that can handle the complex problems that we face today and in the future.

For example, if the origins of life are to be explained, biologists and mathematicians need to work together, and most of the time spent will be on evaluating and simulating the mathematical models ${ }^{2}$. Using the mathematics of evolutionary dynamics, the change from no life to life (referring to the self-replicating molecules dominating early Earth) can be explained. Another example is the electronics industry, which all of us rely on for new developments in virtually every aspect of our everyday life. Innovations in this branch of industry are impossible without the use of virtual design environments that enable engineers to develop and test their complex designs in front of a computer screen, without ever having to go into the time-consuming (several month long) process of prototyping.

Principles of computational science and engineering rooted in modern applied mathematics are at the core of these developments and represent subjects that are set to undergo a renaissance in the 21 st century. Indeed,

\footnotetext{
2 The statement "Biology is the new physics" is heard frequently nowadays; see the EMBO report by Philip Hunter (2010).
} 
no less a figure than Stephen Hawking is on record as having said that the 21st century will be the century of complexity. Another great figure, still young, is Fields medallist Terence Tao, who was a major contributor to the document entitled "The mathematical sciences in 2025" [1], stating: "Mathematical sciences work is becoming an increasingly integral and essential component of a growing array of areas of investigation in biology, medicine, social sciences, business, advanced design, climate, finance, advanced materials, and many more - crucial to economic growth and societal well-being". The recent report by Philip Bond entitled "The era of mathematics" [2] is also a source of inspiration for mathematicians and has led to much additional funding for mathematics in the UK.

Growing computing power, nowadays including multicore architectures and GPUs, does not provide the solution to the ever-growing demand for more complex and more realistic simulations. In fact, it has been demonstrated that Moore's Law, describing the advances in computing power over the last 40 years, holds equally for mathematical algorithms. Hence, it is important to develop both faster computers and faster algorithms at the same time. This is essential if we wish to keep up with the growing demands by science and technology for more complex simulations. For this reason, we have recently introduced the terminology "mathware" 3 to distinguish mathematical method development from software and hardware activities. It is essential that all 3 disciplines cooperate closely, as mathware methodologies may have consequences for hardware, just as hardware has consequences for the development of mathematical methods (Figure 1).

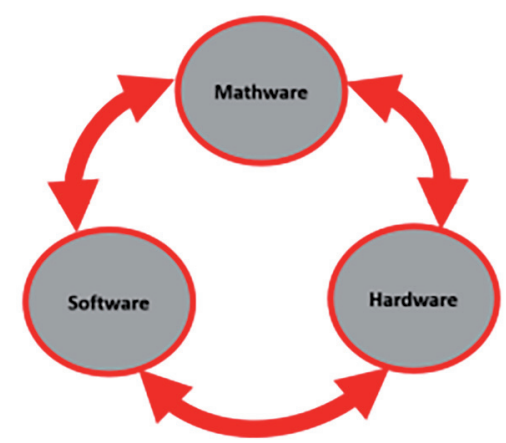

Fig. 1. The development of mathematical methods ("mathware") is an activity distinct from software and hardware. Close cooperation between the three disciplines is of the utmost importance.

Given the above developments, Europe has launched many initiatives to convince industry, society and policymakers that the time is ripe for change. After the OECD report (2008), initiated and chaired by Willi Jaeger from Heidelberg, the European Mathematical Society and the European Science Foundation funded a so-called For-

\footnotetext{
3 The terminology "mathware" was first introduced by the Laboratory for Industrial Mathematics Eindhoven (LIME - https://www.mathware.nl/), but has been adopted by EUMATHS-IN.
}

ward Look project on "Mathematics in Industry". The result of this project was a report with recommendations to policymakers, industry and the mathematics community, and a very nice book European success stories in industrial mathematics, containing more than 100 industrial cases in which mathematics played a decisive role. In 2012, this was followed by a report by Deloitte (accountants and advisers) on "The value of the mathematical sciences for industry and society in the UK", revealing that 38 percent of GVA of the UK can be attributed to results of mathematical sciences research, in a direct, indirect or induced way. Similar studies with comparable conclusions have been undertaken in The Netherlands (2014), France (2017) and Spain (2019) ${ }^{4}$. In Germany, a book was published entitled Mathematics, engine of the economy (2008) with more than 20 accounts by captains of industry, emphasising the importance of mathematics. This shows that Europe is putting a lot of effort into demonstrating the necessity and indispensability of mathematics for industry and society.

In this paper, these initiatives will be discussed, as well as the strategy adopted in Europe. All of these efforts have culminated in the formation of a new foundation called EU-MATHS-IN, that aims at collecting all national and European initiatives in the area of industrial mathematics, so as to learn from each other, to share best practice, and to benefit from a unified approach.

\section{The European Consortium for Mathematics in Industry}

From a historical point of view, ECMI, the European Consortium for Mathematics in Industry [3], was one of the first organisations that was founded to foster the potential of applications of mathematics in industry. It celebrated its 25th anniversary in 2012. Back when it started, in the middle of the 1980s, mathematics was dominated by mathematicians mainly interested in pure mathematics, in algebra, topology, geometry, analysis and so on. Only a small group of people focussed their attention on cooperation with industry. In 1985, this led to the first conference, called the European Symposium for Mathematics in Industry (ESMI). After this successful symposium, it was felt that it would be good to start a European organisation, and hence, in 1987, ECMI was founded. The goal was to promote and further the effective use of mathematics as well as closely related knowledge and expertise in industrial or management settings. More specifically, concerning research, to see what is needed by industry and commerce, to assess what is available, and to discuss what can be done to fill the gaps. Also, it was important to encourage the participating organisations to have joint research ventures. From an educational point of view, the focus was on the creation, organisation and quality control of a 2-year postgraduate course on industrial and management mathematics. It was also decided to have an annual conference. Quite quickly this became a biennial conference focussing

4 All of these reports can be found on the website of EUMATHS-IN: https://www.eu-maths-in.eu. 
on applications of mathematics in industry. The ECMI Newsletter of October 2012 includes a very nice account by one of the founding fathers of ECMI, Helmut Neunzert, about the start and the first 10 years of ECMI. Below is a copy of the official list of signatures on the founding document.

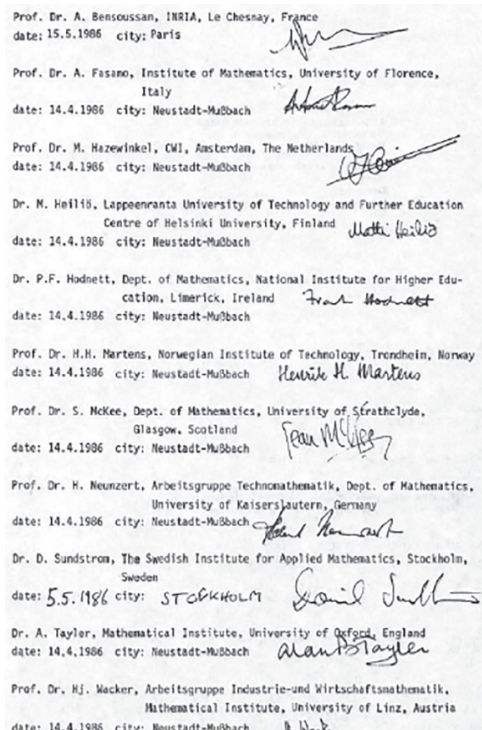

Fig. 2. The founding fathers of ECMI.

ECMI is now a mature organisation with over 30 years of experience in the area of mathematics for industry. Its mission can be summarised as follows:

Mathematics, as the universal language of the sciences, plays a key role in technology, economics and life sciences. European industry is increasingly dependent on mathematical expertise in both research and development to keep its world-leading role for high technology innovations and to comply with the EU 2020 agenda for smart, sustainable and inclusive growth. The major objectives to respond to these needs of European industry may be summarized as follows:

\section{- ECMI advocates the use of mathematical models in industry \\ - ECMI stimulates the education of young scientists to meet the growing demands of industry \\ - ECMI promotes European collaboration, interaction and exchange within academia and industry}

One of the most successful enterprises of ECMI is in the educational field. Its Educational Committee consists of many experts that meet regularly and discuss curricula for master's degrees in industrial mathematics, as well as keeping a close eye on the quality of such curricula in member universities. This quality control is carried out on a regular basis, new members can apply for the status of qualified node and are then visited by a team of experts evaluating the curriculum and the means used. Nowadays, the ECMI Educational Committee oversees more than 20 high standard master's programs in industrial and econo-mathematics. Students that have graduated from an ECMI centre are awarded an ECMI certificate.
Another one of ECMIs success stories are the annual European Modelling weeks that started in 1988 in Bari (Italy) with 30 students working on six projects. Each project in a modelling week originates from a real-life problem and an international student group, supervised by an ECMI instructor, works collaboratively for one week towards a solution. In 2019, the 34th modelling week took place in Grenoble (France), where some 40 students from all over Europe worked on 7 projects. Alongside the modelling week, ECMI also organises its summer school, where lecturers both from ECMI and from its industrial partners give courses in various topics of applied and industrial mathematics.

An important aspect of the ECMI education network is the organisation and broadening of the exchange of students among the ECMI centres. The strong coherence within the network and the synchronised local master programs taught in English allow for a smooth relocation from one centre to another and for an easy transfer of credits gained at a foreign centre.

ECMI also undertakes many activities in the research area. The Research and Innovation Committee focuses on strategies to increase the interaction between industry and academia, to foster both academic research and industrial innovation. The committee is multidisciplinary. It marshals the power of mathematics, scientific computing and engineering for industrial modelling and simulation. It also fosters special interest groups (SIGs) that focus on a special theme which is either application oriented or methodology based. A SIG identifies a group of experts and has a strong industry participation or interest. The SIGs organise regular meetings and workshops. Examples of active SIGs are "Scientific Computing in the Electronics Industry" and "Shape and Size in Medicine, Biotechnology and Material Sciences". The SIGs provide a unique opportunity for cooperation on the European level, participating jointly in, for example, an Innovative Training Network within the Marie-Sklodowska-Curie program. Two very successful examples of past projects are MACSI-net (Mathematics, Computing and Simulation for Industry) and COMSON (Coupled Multiscale Simulation and Optimization in Nanoelectronics). The former is considered to have been extremely important for the further development of strategies to bridge the gap with industry, and is therefore discussed below in more detail.

Last, but certainly not least, are the European Study Groups with Industry (ESGI). Study Groups with Industry [4] are an internationally recognised method of technology transfer between academic mathematicians and industry. These weeklong workshops provide a forum for industrial scientists to work alongside academic mathematicians on problems of direct industrial relevance. The success of the Study Groups' unique format, which uses problems presented by industry as a basis for mathematical research, is demonstrated by the extent to which it has been copied around the world and is now extending into other areas where mathematics may be applied. The European Study Groups with Industry started with the first Study Group in Oxford in 1968, and now there are 5-7 meetings held annually in different European countries. 


\section{MACSI-net}

ECMI provided the cradle for a very successful European network, initiated by Prof. Bob Mattheij at TU Eindhoven, one of the founding fathers of ECMI. MACSI-net [5], short for Mathematics, Computing and Simulation for Industry, was in fact a cooperative venture between ECMI and ECCOMAS [6]. ECCOMAS is a scientific organisation grouping together European associations with interests in the development and application of computational methods in science and technology. The Mission of ECCOMAS is to promote joint efforts of European universities, research institutes and industries which are active in the broader field of numerical methods and computer simulation in Engineering and Applied Sciences, and to address critical societal and technological problems with particular emphasis on multidisciplinary applications.

When MACSI-net was started around the turn of the century, it was apparent that industry was grappling with ever more challenging problems which should be solved by using state of the art mathematical and computational tools. Academic institutions often had the knowledge and expertise to be of great help with this. However, enterprises often did not know how to find the proper academic partners, in particular in mathematical areas. Equally, academic institutes were still not sufficiently aware of the importance of taking up their role in joint endeavours with both smaller and larger problems that could help Europe's industry to maintain or achieve a competitive edge in a variety of areas. MACSI-net was therefore set up as a network where both enterprises and university institutions could cooperate on the solution of such problems, to their mutual benefit. In particular, the network focused on strategies to increase the interaction between industry and academia in order to help industry (in particular SME) with advanced mathematical and computational tools, and to increase awareness of academia concerning industrial needs. The network was multidisciplinary, combining the power of mathematics, scientific computing and engineering, for modelling and simulation activities. The network aimed at achieving its goals through

- Strategic meetings with industries about well-specified topics

- Summer courses

- Workshops

- Visits of experts

- Foundation of special (interest) groups

- Funding and appointment of post docs

- Activity committees who actively look for funded proposals from EU or other bodies

The various nodes in the MACSI-net network each fostered general and specific expertise in areas of mathematics and computing. The role of industrial nodes was somewhat complementary to the academic ones. The network was aiming at the dissemination of ideas, models and algorithms to their mutual benefit, leading to joint research efforts and the forging of (often thinly spread) local initiatives. In particular, joint research proposals were expected to make this network attractive for all involved.

MACSI-net was very successful during its 4 years of existence. In the end, there were 17 working groups concentrating on a large variety of topics. Some of these working groups are still active, in a different form, but the researchers have remained in contact. An example of this is working group 2 on Coupled problems and Model Order Reduction. It actually split into two different communities, one of these active within ECCOMAS and organising biennial conferences on coupled problems (see, for example, [7]). The other group remained concentrated on model order reduction, and ran the European COST Action EU-MORNET from 2014-2018 which was used to coordinate all research in the area of model order reduction taking place in Europe [8].

At the end of its lifetime, in 2004, MACSI-net issued an important document which was one of the first reports on industrial mathematics with guidelines and recommendations. Some quotes from this document:

- Mathematics should be regarded as a technology in its own right. Its crucial role in many industrial problems requires the active participation of mathematicians. Truly multidisciplinary projects will benefit significantly from the involvement of mathematical modellers and this should be encouraged by future funding programmes. Consideration should be given to making the participation of mathematicians in appropriate multidisciplinary projects a condition of project funding.

- There is a need for positive action to promote the increased use of mathematics by European industry. The success of local initiatives where mathematicians are working on industrially relevant problems is clear evidence that they are already making a significant contribution to the development of the knowledgebased economy. However, more needs to be done to encourage companies, especially Small and Mediumsized Enterprises (SMEs), to make use of mathematics and mathematicians. Consideration should be given to creating a programme funding projects that will enable companies, especially SMEs, to explore areas where mathematics can make a contribution to their improved competitiveness.

- There is an urgent need for more training in the area of industrial mathematics. It is essential to attract bright students to this area and to convey the challenge and the excitement of solving practical problems. Consideration should be given to specific funding for training programmes in industrial mathematics across Europe.

The full report can be found here [9]. MACSI-net ended in 2004, but the acronym is in fact still in use. At Limerick University, Prof. Stephen O'Brien attracted funding from the Science Foundation Ireland and is running a project termed MACSI [10], which is a network of mathematical modellers and scientific computational analysts based in Ireland. Its aim is to foster new collaborative research, in 
particular on problems that arise in industry, in order to produce world-class publications on mathematical modelling. It has been very successful to date, attracting a lot of Irish industry.

\section{A renowned institute for mathematics and industry}

One of the founding fathers of ECMI, already mentioned earlier, was Prof. Helmut Neunzert, who was also the key driving force behind the creation and subsequent success of the Fraunhofer Institute for Industrial Mathematics (ITWM) that started in Kaiserslautern in the middle of the 1990s. On their website [11], one can find the following remark which encapsulates the essence of the role and importance of industrial mathematics:

\begin{abstract}
"The core competence of ITWM is mathematics: the language used by scientists and engineers to formulate models for technical systems. In our time it is particularly important, as it provides efficient algorithms to compute and analyse such models. The ITWM's mission is to develop this technology to give innovative impulses and put them into practice together with industry partners. Since its foundation in 1995 the ITWM has shown great success in building mathematical bridges between applied sciences and concrete application. Clients are large international companies as well as small and medium regional enterprises. Fraunhofer ITWM focuses on the development of mathematical applications for industry, technology and economy. Mathematical approaches to practical challenges are the specific competences of the institute and complement knowledge in engineering and economics in an optimal way. In 2001 ITWM became the first mathematical oriented institute of the Fraunhofer Gesellschaft. The main emphases are surface quality inspection, financial mathematics, visualization of large data sets, and optimization of production processes, virtual material design and analysis of $3 D$ models of microstructures."
\end{abstract}

ITWM is an example of how mathematics can successfully be turned into a business. Since ITWM's foundation, its budget has increased substantially: beginning with 1,64 million $€$ in 1995 , it reached 31.4 million $€$ in 2019. Nearly 75 percent of the operating budget stems from the institute's own profits. At present, ITWM's personnel consists of almost 500 employees, of which 160 are PhD students.

The success of ITWM has also been observed by others, and by now there are various smaller and larger companies that obtain their business from the application of mathematics to industrial problems. An example is the Laboratory for Industrial Mathematics Eindhoven (LIME) [10] in the Netherlands that originally started at the Eindhoven University of Technology, but soon after became an independent company.

\section{Captains of industry reporting on mathematics}

The book Mathematik - Motor der Wirtschaft [12] came about in close cooperation between the Oberwolfach
Foundation and the Mathematisches Forschungsinstitut Oberwolfach, and features articles by renowned business figures. It was launched by the German Federal Minister of Education and Research, Annette Schavan, at a gala event. Oberwolfach is well known for its workshops on mathematics, but this event was a very special one, involving many captains of industry outlining their opinions about mathematics and its utilisation in their companies.

In their articles, various heads of major German companies - Allianz, Daimler, Lufthansa, Linde, and TUI, to name but a few - sum it up in a nutshell: mathematics is everywhere, and our economy would not work without it. SAP's CEO, Henning Kagermann, puts it like this: "Corporate management without mathematics is like space travel without physics. Numbers aren't the be all and end all in business life. But without mathematics, we would be nothing."

\section{The OECD report on mathematics and industry}

While ECMI continued to attract new members and spread its activities further across Europe, also including countries in the eastern part, the idea arose in Heidelberg to use the experience gained in several countries to start a series of discussions and produce a report on mathematics for industry. To this end, the initiator, Prof. Willi Jäger, who heads the institute IWR (Interdisciplinary Center for Scientific Computing) [13], suggested the idea to the OECD.

Recognising the importance of mathematics in an industrial context, the delegates to the Global Science Forum (GSF) of the Organization for Economic Cooperation and Development (OECD) agreed to sponsor an international consultation to assess the present state of this interface in the participating countries and to identify mechanisms for strengthening the connection between mathematics and industry. (The interaction between mathematics and other sciences was left for future consideration.)

A workshop on "Mathematics in Industry" was then held in Heidelberg in early 2007. The objectives of the workshop were to

- analyse the relationship between the mathematical sciences and industry in the participating countries;

- identify significant trends in research in the mathematical sciences in academia and the mathematical challenges faced by industry in the globalised economic environment, and to analyse the implications of the trends for the relationships between mathematical scientists in academia and industry;

- identify and analyse major challenges and opportunities for a mutually beneficial partnership between industry and academia; and

- formulate action-oriented practical recommendations for the main stakeholders: the community of mathematical scientists, participating industries and governments.

The report [14] summarised the deliberations and presented the findings and recommendations of the work- 
shop, which will involve further consultations among the participants. The recommendations involved the participation of the academic community, governmental and other funding agencies as well as industry. They were designed to stimulate the interaction between mathematics and industry; to enhance the curriculum for students of mathematics; to improve the infrastructure for increased interaction, both in academia and in industry; and to strengthen coordination and cooperation at national and international levels.

As a follow-up, the OECD also supported an activity that was intended as a corollary to the report "Mathematics-in-Industry". It primarily comprises a factual compendium of the ways in which the various mechanisms cited in that report have been implemented around the world. The compendium, which is not comprehensive, has been compiled with the aim of helping governments, industries and academia to see how they may best exploit mathematics as an industrial resource for both research and training.

\section{The forward look initiative of ESF and EMS}

Although the reports commissioned by the OECD were a very valuable asset to the mathematics community, it was felt that an even more in-depth understanding of the problems was necessary. Indeed, the impact of mathematics on industry and society had been the subject of numerous studies, but it was decided at the end of 2009 to start a Forward Look project on mathematics and industry, evolving from the belief that European Mathematics as a whole has the potential to boost European knowledge-based innovation, which is essential for a globally competitive economy. The project was fostered by the European Science Foundation and the European Mathematical Society, and involved many members of ECMI, as it was felt they had the experience and knowledge to be able to implement such an activity. The main driving force for this endeavor was Prof. Mario Primicerio from Firenze University.

The Forward Look at mathematics and industry sprung from the strong belief that European Mathematics has the potential to be an important economic resource for European industry, helping its innovation and hence its capacity for competing on the global market. To fulfil its potential, special attention has to be paid to the reduction of the geographical and scientific fragmentation in the European Research Area. Overcoming this fragmentation will require the involvement of the entire scientific community. Europe needs to combine all experience and synergies at the interface between mathematics and industry and create strong areas of interaction to turn challenges into new opportunities.

The project began in 2009, and working groups were set up to discuss the main issues identified. An extensive survey was carried out to identify whether the topics worked on in academic circles reflected the needs of industry. In the figure below, this is illustrated. It confirms that, apart from a very small number of exceptions, mathematicians are indeed doing valuable work in areas of importance in industry.

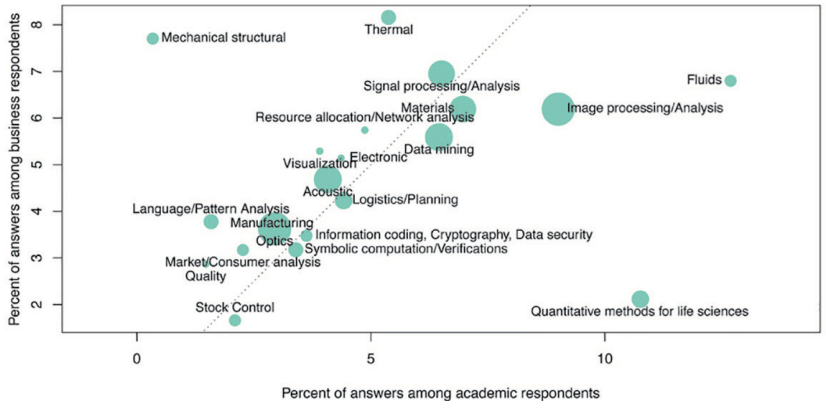

Fig. 3. Main areas of competence available in academia versus major business challenges perceived by industry (size of the bubbles indicates total number of respondents).

The project also organised alignment and consensus conferences, involving many researchers and industrialists from all over Europe, so that the conclusions in the final report [15] were broadly supported and adopted. The final recommendations were:

- Recommendation 1: Policymakers and funding organisations should join their efforts to fund mathematics activities through a European Institute of Mathematics for Innovation.

- Recommendation 2: In order to overcome geographical and scientific fragmentation, academic institutions and industry must share and disseminate best practices across Europe and disciplines via networks and digital means.

- Recommendation 3: Mathematical Societies and academic institutions should create common curricula and educational programmes in mathematics at the European level taking into account local expertise and specificity.

Besides these recommendations, the report also gives roadmaps for their implementation.

\section{The network of networks EU-MATHS-IN}

Even though the recommendations from the aforementioned Forward Look report were widely accepted, it turned out to be quite hard to obtain sufficient support to implement them in practice. Therefore, in 2013, it was decided to take the initiative into our own (mathematical) hands and start a new organisation in Europe that would enable cross-fertilisation and exchange of best practice. Collaboration provides a much better basis for funding of European organisations. Consequently, at the end of 2013, EU-MATHS-IN was launched in Amsterdam [16]. It is the European Service Network of Mathematics for Industry and Innovation. As stated on the website: "A new initiative to boost mathematics for industry in Europe. Make the most of our expertise for a more efficient route to innovation!"

EU-MATHS-IN aims to leverage the impact of mathematics on innovations in key technologies through enhanced communication and information exchange between and among the involved stakeholders at a European level. It aims to create a dedicated one-stop shop, 
together with other stakeholders, to coordinate and facilitate the required exchanges in the field of applicationdriven mathematical research and its exploitation for innovations in industry, science and society. For this, it aims to build an e-infrastructure that provides tailored access to information and facilitates communication and exchange by player-specific sets of services. It will act as facilitator, translator, educator and link between and among the various players and their communities in Europe. In the figure below, a graphical illustration is given of the intended structure.

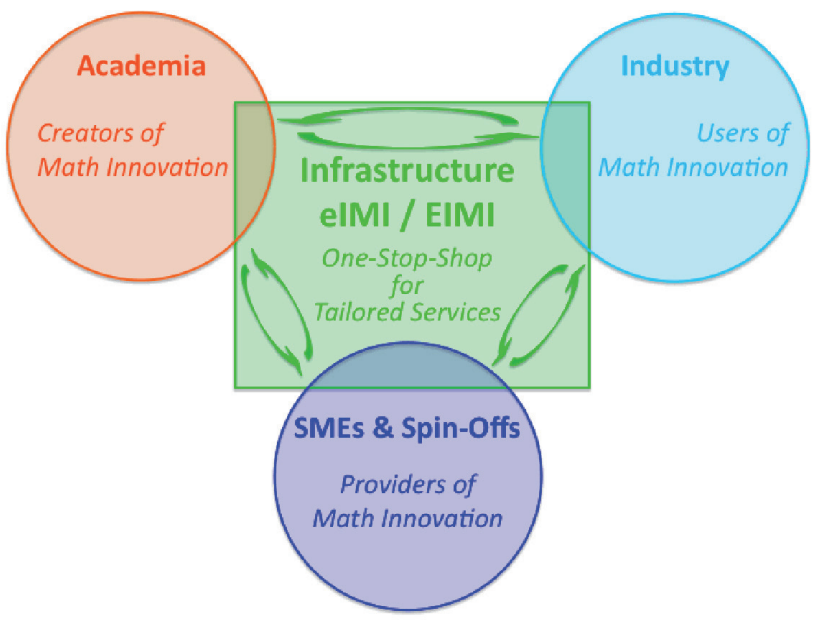

Fig. 4. Graphical illustration of the strategy of EU-MATHS-IN.

The important features of the long-term goals of the organisation are:

- Establish strategic connections among the national networks and centres working in the field of industrial mathematics and mathematics for innovation;

- Create a European service unit that can foster the competitive advantage of the European industry through international cooperation;

- Promote the technological aspects of mathematics raising public awareness;

- Stimulate the cooperation at European level of mathematical research with companies and administrations;

- Establish a one-stop-shop at European level for industrial users of mathematical scientific research results;

- Provide European industry, in particular SMEs, with a competitive advantage taking profit of the scientific excellence of the continent (give Europe the possibility to cash a "scientific dividend");

- Acquire funding for the performance of activities that serve the realisation of the Association's aims.

It is felt that, with EU-MATHS-IN, Europe has a powerful organisation that will be able to bring together all national initiatives, such as those that have arisen in many European countries, to learn from each other, to share experiences and together form a community that is recognised for its capability to bridge the gap between mathematics and industry.

One of the first initiatives of the organisation was to strongly call for the establishment of Mathematical Mod- elling, Simulation and Optimization (MSO) as a transversal (universal?) Key Enabling Technology (KET). The arguments are as follows. There is no doubt that continuous multidisciplinary research and novel mathematical and computational methods are needed to provide the necessary tools for industrial innovation and European competitiveness. It has become widely recognised that the approach of MSO is the third, and indispensable, pillar for scientific progress and technological innovation, alongside experiments and theory building. Experience shows that future challenges for innovation in industry and society will involve increasing complexity and, at the same time, are subject to ever-shorter innovation cycles. The real-world challenges to be dealt with on our way towards innovation exhibit opportunities that make MSO indispensable and simultaneously a far from trivial task.

In 2017, EU-MATHS-IN invited a number of European companies to actively join in the discussions to promote mathematics for industry in Europe. The Industrial Core Committee (ICC) was formed, currently with Siemens, Michelin, Shell, Bosch, ATOS, Nors, Repsol and ING on board. The formation of the ICC was instrumental for EU-MATHS-IN: two workshops were held in Amsterdam, and a workshop on "Future and emerging mathematical technologies in Europe" was held in the Lorentz Center in Leiden at the end of 2017 [17]. These events led to a vision document entitled "Modelling, Simulation \& Optimization in a Data rich Environment - A window of opportunity to boost innovations in Europe" [18] that was presented in the French embassy in Berlin on 18 April 2018. The document contains the joint vision on MSO in a data rich environment. A delegation of EUMATHS-IN and its ICC visited the unofficial opening event of the new joint undertaking EuroHPC in Sofia [19], and used this opportunity to speak to the (then) European Commissioner for Digital Economy and Society, Marya Gabriel. Mathematics is an important ingredient for EuroHPC, and to make this apparent, a separate document was produced by the ICC (see the web page of EU-MATHS-IN).

Realising that it is important to gather arguments in order to convince policymakers, EU-MATHS-IN set out to write its first Strategic Research Agenda (SRA) in 2019. Vice-president of EU-MATHS-IN, Zoltan Horvath, is leading this effort. There were 9 working groups started, on basic MSODE technologies, 4 working groups on missions (one of them being digital twins) and 1 working group on transfer. Due to the COVID-19 crisis, the work on the SRA has been delayed significantly, but it is anticipated that the final SRA will be published in early 2021. We feel that it will be an extremely important document in discussions with policymakers, firmly backed by the ICC and other European industry.

As a corollary of the SRA efforts, EU-MATHS-IN recently got involved in the TransContinuum Initiative (TCI), initiated by the organisations ETP4HPC and BDVA, set up in the advent of the new Horizon Europe program. The term TransContinuum describes the defining characteristic of the infrastructure required for the 
convergence of data and compute capabilities in many leading edge industrial and scientific use scenarios. The initiative outlines a vision for a horizontal collaboration between European associations and projects involved in IT technology, application and services provisioning for the Digital Continuum. Mathematical Models and Algorithms are in the kernel of the activities: TCI describes a continuous dynamic workflow between smart sensors and IOT devices at the edge and HPC/cloud centres over smart networks and services executing simulation \& modelling, big data analytics and machine learning, based on mathematical methods and algorithms including MSODE, pervasively augmented by artificial intelligence, protected and secured by cybersecurity and back to cyber-physical systems. We feel that such joint undertakings are extremely important for mathematics, being part of the game that is being played.

In order to convince policymakers that mathematics is indispensable in today's world, we are also working on a pamphlet entitled "Horizon Europe needs Mathematics". This pamphlet will contain a few one-liners stressing the importance of mathematics. The statement by Lex Schrijver ("mathematics is like oxygen") will be one of these, as will "Mathematics: invisible contribution to visible success" and "Mathematics: real intelligence is needed to make artificial intelligence work. In addition, we will argue that mathematical algorithm development has outperformed machine improvement ("Moore's Law") in the past 4 decades. Finally, the following statement made in the report "The era of mathematics" [2] provides a very strong argument: "The rate of return on investment as benefit-to-cost ratio may be estimated as follows: Engineering 88, Physics 31, Chemistry 246, and Mathematical Sciences 588." The pamphlet will be made available on the website of EUMATHS-IN [16].

\section{Industrial mathematics on the world scale}

Europe has always been very active in building bridges between mathematicians and industry. But also in other continents, and on the world scale, similar initiatives and organisations have emerged. The largest association is the Society for Industrial and Applied Mathematics (SIAM), based in the USA, but also operating more widely. SIAM was already founded back in 1951, and many researchers worldwide are members of this organisation.

In 1986 the four societies GAMM, IMA, SIAM and SMAI decided to organise large International Conferences on Industrial and Applied Mathematics (ICIAM) every four years. The first of such conferences was held in Paris in 1987, and since then it has been organised on different continents every 4 years, the latest edition in Valencia with over 4000 participants. A great account of the history was written by Iain Duff in 2007 [20]. Currently, ICIAM has over 50 members, and growing. We feel that it is very important to share the experience we have in Europe with the rest of the world, so as to make sure that mathematics is recognised everywhere as a key enabling technology that should be firmly adopted when working on mathematics for industry.

\section{Conclusion}

Europe has always been very active in trying to bridge the gap between mathematics and industry. Already since the 1970s, when the Oxford study groups with mathematics started to be held, mathematicians have realised the potential for breakthroughs and innovations in industry and for societal problems. In this paper, we have given a chronological picture of what has happened in Europe since the 1970s. We are observing a very natural and continuous growth of activities, stepping up in intensity over the years. In recent years, a strong linking of mathematicians and mathematics with industry personnel and problems has been occurring, with the importance of industrial mathematics being realised. This is confirmed by recent reports issued on the economic value of mathematics, in various European countries, concluding that about 30 percent of GVA can be attributed to the results of research in the mathematical sciences. This is an enormously large percentage, and it will hopefully convince politicians and policymakers to invest more in mathematics.

The foregoing leads to the natural question: what will our world look like in 2030? And what will the role of the mathematical sciences be in shaping that world? Since the start of the 21st century, it has become clear that the mathematical sciences are gaining a new stature. They are increasingly providing the knowledge to enable innovative breakthroughs and insights in many other disciplines such as biology, healthcare, social sciences and climatology, alongside their traditional role in physics, chemistry and computer science. The importance of the mathematical sciences is also rapidly increasing in the business world, for example in design processes, electronics and finance. All these developments are vital for economic growth and competitive strength, and demand an in-depth review of the overall way we look at the mathematical sciences. This involves the integration of mathematics with statistics, operations research and computational science, and it carries implications for the nature and scale of research funding.

Joining forces is very important in this endeavour, as is using the right kind of PR. To this end, EU-MATHSIN has developed the "Mathematics Inside" pictogram that can be used to convey the message that mathematics is indispensable and present everywhere. Dutch mathematician Lex Schrijver formulated this in a very succinct way: "Mathematics is like oxygen. You take no notice of it when it's there - if it wasn't, you'd realize you cannot do without it." The pictogram below allows us to spread this message!

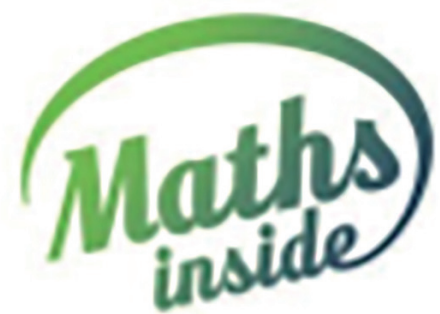




\section{References}

[1] https://www.nap.edu/catalog/15269/the-mathematical-sciencesin-2025

[2] https://epsrc.ukri.org/newsevents/pubs/era-of-maths/

[3] http://www.ecmi-indmath.org

[4] https://ecmiindmath.org/study-groups/

[5] http://www.macsinet.org

[6] http://www.eccomas.org

[7] https://congress.cimne.com/coupled2019/frontal/Series.asp

[8] http://www.eu-mor.net/

[9] http://www.mcs-uab.com/docs/MACSINET.Roadmap_for_ Mathematics_in_European_Industry-1.2004.pdf

[10] https://ulsites.ul.ie/macsi/

[11] https://www.itwm.fraunhofer.de/

[12] https://www.springer.com/gp/book/9783540786672

[13] https://typo.iwr.uni-heidelberg.de/home/

[14] https://www.eu-maths-in.eu/wp-content/uploads/2016/02/2008_ EU_OECD_MathsinIndustry.pdf

[15] http://www.eu-maths-in.eu/reports/

[16] https://www.eu-maths-in.eu

[17] https://www.lorentzcenter.nl/future-and-emerging-mathematicaltechnologies-in-europe.html
[18] https://www.eu-maths-in.eu/wp-content/uploads/2018/05/MSOvision.pdf

[19] https://ec.europa.eu/digital-single-market/en/news/shapingeuropes-digital-future-bulgarian-presidency-event-high-performance-computing

[20] http://www.iciam.org/start-iciam-congresses-iain-duff

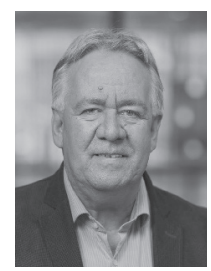

Wil Schilders (MSc Nijmegen 1978, PhD Trinity College Dublin 1980; https://www. win.tue.nl/ wschilde/). In industry (Philips, NXP Semiconductors) from 1980 to 2010. Since 1999 he has been a professor of scientific computing for industry at Eindhoven University of Technology. He was president of ECMI (2010-2011), and is co-founder and current president of EU-MATHS-IN. He is also the director of the Dutch Platform for Mathematics and was elected officer at large on the board of ICIAM in 2019. 